\title{
Article \\ Detection of Del/Dup Inside SHOX/PAR1 Region in Children and Young Adults with Idiopathic Short Stature
}

\author{
Jera Stritar ${ }^{1}$, Lana Stavber ${ }^{2}$, Maja Ficko ${ }^{1}$, Primož Kotnik ${ }^{2}$, Tadej Battelino ${ }^{2,3}$, Katarina Trebušak Podkrajšek ${ }^{1,4}$ (i) \\ and Tinka Hovnik $1,4, *$ \\ 1 Clinical Institute of Special Laboratory Diagnostics, University Children's Hospital, UMC, 1000 Ljubljana, \\ Slovenia; jera.stritar@kclj.si (J.S.); maja.ficko@kclj.si (M.F.); katarina.trebusakpodkrajsek@mf.uni-lj.si (K.T.P.) \\ 2 Department of Pediatric Endocrinology, Diabetes and Metabolic Diseases, University Children's Hospital, \\ UMC, 1000 Ljubljana, Slovenia; lana.stavber@kclj.si (L.S.); primoz.kotnik@kclj.si (P.K.); \\ tadej.battelino@kclj.si (T.B.) \\ 3 Department of Pediatrics, Faculty of Medicine, University of Ljubljana, 1000 Ljubljana, Slovenia \\ 4 Institute of Biochemistry and Molecular Genetics, Faculty of Medicine, University of Ljubljana, \\ 1000 Ljubljana, Slovenia \\ * Correspondence: tinka.hovnik@kclj.si
}

Citation: Stritar, J.; Stavber, L.; Ficko, M.; Kotnik, P.; Battelino, T.; Trebušak Podkrajšek, K.; Hovnik, T. Detection of Del/Dup Inside SHOX/PAR1 Region in Children and Young Adults with Idiopathic Short Stature. Genes 2021, 12, 1546. https://doi.org/ 10.3390/genes12101546

Academic Editor: Olaf Bodamer

Received: 31 August 2021

Accepted: 27 September 2021

Published: 29 September 2021

Publisher's Note: MDPI stays neutral with regard to jurisdictional claims in published maps and institutional affiliations.

Copyright: (c) 2021 by the authors. Licensee MDPI, Basel, Switzerland. This article is an open access article distributed under the terms and conditions of the Creative Commons Attribution (CC BY) license (https:/ / creativecommons.org/licenses/by/ $4.0 /)$.
Abstract: Short stature is a common growth disorder defined as a body height two standard deviations (SD) or more below the mean for a given age, gender, and population. A large part of the cases remains unexplained and is referred to as having idiopathic short stature (ISS). One of the leading genetic causes of short stature is variants of short stature homeobox-containing gene (SHOX) and is considered to be responsible for $2-15 \%$ of ISS. We aimed to analyse the regulatory and coding region of SHOX in Slovenian children and young adults with ISS and to investigate the pathogenicity of detected variants. Our cohort included 75 children and young adults with ISS. Multiplex ligation-dependent probe amplification (MLPA) was performed in all participants for the detection of larger copy number variations (CNVs). Sanger sequencing was undertaken for the detection of point variants, small deletions, and insertions. A total of one deletion and two duplications were discovered using the MLPA technique. Only one of these four variants was identified as disease-causing and occurred in one individual, which represents $1.3 \%$ of the cohort. With Sanger sequencing, two variants were discovered, but none of them appeared to have a pathogenic effect on height. According to the results, in the Slovenian population of children and young adults with ISS, $S H O X$ deficiency is less frequent than expected considering existing data from other populations.

Keywords: height; short stature; SHOX; MLPA; Sanger sequencing

\section{Introduction}

Short stature is a frequent developmental condition in childhood and is one of the most common reasons for visiting paediatric endocrinologists. The prevailing pathological causes of short stature are growth hormone $(\mathrm{GH})$ deficiency, genetic disorders, hypothyroidism, and celiac disease. However, in 50-90\% of the cases, no obvious pathological cause is found, so they are classified in the group of normal short stature-familial short stature, constitutional delay of growth and puberty, or idiopathic short stature (ISS). It is difficult to distinguish these three populations as they have multiple overlapping features; thus, they all could be considered to have idiopathic short stature. Short stature is also characteristic for infants born small for gestational age (SGA), the majority of which have catch-up growth in the first year of life [1,2].

According to the Consensus Statement from 2008, ISS is defined as a height two standard deviations (SD) or more below the corresponding mean height for a given age, sex, and population group without evidence of systemic, endocrine (e.g., GH deficiency, hypothyroidism, Cushing syndrome, precocious puberty), nutritional, chromosomal abnormalities (e.g., Turner syndrome), evident skeletal dysplasia, or other genetic syndromes 
(Noonan, Silver-Russell, Prader-Willi) [3]. ISS diagnosis is complex and based on medical history, physical examination, standard laboratory tests, and genetic analysis. There is still a large proportion of those in whom the specific genetic cause by standard and existing genetic analysis remains undetermined.

Processes involved in growth can be interrupted by genetic, epigenetic, and environmental factors [4]. Moreover, it is well known that height is one of the human polygenic traits with the highest degree of heritability (60-80\%) [1]. Genome-wide association studies (GWAS) revealed that the accumulation of multiple genetic variants (i.e., single nucleotide polymorphisms (SNP)) affects growth, but each contributes a very small proportion to a final height [4]. However, in some individuals reduced stature is caused by a specific genetic change with a large effect, such as variants in the regulatory and coding region of the short stature homeobox-containing gene (SHOX) that are supposedly responsible for $2-15 \%$ of short stature, formerly diagnosed as idiopathic [1,5-7].

The SHOX gene is located in the telomeric pseudoautosomal region 1 (PAR1) on the short arm of sex chromosomes and plays an important role in the skeletal development of vertebrates. It escapes the inactivation of the $\mathrm{X}$ chromosome and consequently remains expressed on both sex chromosomes. Even though the SHOX gene is located on sex chromosomes, it is inherited in an autosomal dominant manner $[6,8]$. The most common genetic variants in the SHOX gene are deletions, followed by duplications and point variants [9].

SHOX haploinsufficiency is expressed by a variable clinical phenotype, from extremely short stature to mild short stature with no additional clinical features [10]. The most severe phenotype manifests as Langer mesomelic dysplasia, where both copies of SHOX are lost. Skeletal deformity with severe short stature, mesomelia, and Madelung deformity is characteristic. A milder form of skeletal dysplasia is present in Leri-Weill dyschondrosteosis (LWD), resulting from a heterozygous variant in the SHOX gene. The same phenotype is expressed in Turner syndrome, where the entire $X$ chromosome is lost along with one copy of the SHOX gene. Heterozygous variants of SHOX were also found in individuals with nonsyndromic short stature [11].

This study aimed to assess the frequency of genetic variants in the regulatory and coding regions of the SHOX gene in a group of children and young adults with ISS. In our cohort study, a mutational analysis was performed by multiplex ligation-dependent probe amplification (MLPA) for detecting larger copy number variations (CNVs), followed by Sanger sequencing to determine point variants, small deletions, and insertions.

\section{Materials and Methods}

\subsection{Participants}

The cohort included 75 subjects ( 39 female, 36 male), aged 2 to 22 years old, referred to the University Children's Hospital of Ljubljana between 2015 and 2019. All participants were selected following strict inclusion criteria (i.e., height below $-2 \mathrm{SD}$ ) and exclusion criteria-GH deficiency, chronic systemic diseases (cystic fibrosis, celiac disease, chronic inflammatory bowel disease, type 1 diabetes, etc.), endocrine disorders (Cushing's syndrome, hypothyroidism), defined skeletal dysplasia, chromosomal abnormalities (Turner syndrome, Down syndrome) and growth influencing medications (glucocorticoids) [12]. Arginine and L-Dopa GH stimulation tests were performed according to previously published test procedures [13]. Serum GH levels were determined by immunoassay using Immulite 2000 (Siemens). Bone age was evaluated based on Greulich and Pyle Atlas (GP) bone age determination system, 2nd edition, or determined with the BoneXpert program [14]. Z-scores for height were calculated using the LMS method (L (curve Box-Cox), $\mathrm{M}$ (curve median) and S (curve coefficient of variation)) and the British 1990 reference growth data [15]. Detailed patients' features are given in Supplementary File S1.

The study was conducted according to the guidelines of the Declaration of Helsinki and approved by the Slovene Medical Ethics Committee (0120-36/2019/4). All participants or their legal guardians provided written informed consent before the study. 


\subsection{Methods}

Whole blood EDTA samples were collected. Isolation of genomic DNA was performed using a commercial FlexiGene DNA isolation kit (Qiagen, Hilden, Germany) according to the manufacturer's instructions [16]. The concentration of the isolated DNA was determined using a UV-VIS spectrophotometer. Samples of isolated DNA were stored at $4{ }^{\circ} \mathrm{C}$ throughout the study. All samples were first analysed by the MLPA assay to discover larger CNVs inside the coding sequence of the SHOX gene and enhancer regions in PAR1. Samples in which no change was detected by the MLPA method were further analysed by sequencing all exons in the SHOX gene coding region.

MLPA analysis was performed using the commercial SALSA MLPA Probemix P018-G2 SHOX kit (MRC-Holland, Amsterdam, The Netherlands) according to the manufacturer's instructions. It contains a total of 48 MLPA probes with amplification products between 124 and 504 nucleotides. Thirty-two of these probes are for the PAR1 region on chromosome Xp22/Yp11. Several other probes are included for SHOX regulatory regions upstream and downstream of the gene, and 13 flanking probes in the probemix are targeting the $\mathrm{X}$ chromosome outside the SHOX area. In addition, probemix also contains nine reference probes and ten quality control fragments $[17,18]$. Polymerase chain reaction (PCR) fragments were separated by genetic analyser ABI 3500 (Applied Biosystems, Waltham, MA, USA). The MLPA results were analysed using Coffalyser.Net software [19]. No DNA control and at least three reference DNA samples were included in each MLPA reaction. The results were presented as probe ratios, with the normal number of copies expressed as the ratio of $0.80-1.20$, whereas deletions and duplications were expressed as a ratio less than 0.65 (loss) or greater than 1.30 (gain), respectively. Each detected alternation was confirmed by an independent MLPA reaction.

In Sanger sequencing analysis the SHOX coding region was amplified by PCR, using in-house designed sets of primers. Amplification was performed with an initial step at $95^{\circ} \mathrm{C}$ for $2 \mathrm{~min}$, followed by 35 cycles at $95^{\circ} \mathrm{C}$ for $30 \mathrm{~s}, 64^{\circ} \mathrm{C}$ for $30 \mathrm{~s}$ and $72{ }^{\circ} \mathrm{C}$ for $40 \mathrm{~s}$, and the final extension at $72{ }^{\circ} \mathrm{C}$ for $7 \mathrm{~min}$. After agarose electrophoresis and purification of the amplicons, sequence analysis was performed using BigDye Terminator v3.1 Cycle Sequencing Kit, followed by capillary electrophoresis with 3500 Genetic Analyser (Applied Biosystems, Waltham, MA, USA). The final results were analysed using the BLAST program from the NCBI browser [20], which shows any discrepancies between the sample and control reference sequences. The first exon of the SHOX gene is noncoding; therefore, it was not included in the sequence analysis.

\section{Results}

Our study group comprised 75 participants ( $52 \%$ female) with a mean age of 10.6 years. The mean height was $-2.8 \mathrm{SD}$ for girls and $-2.6 \mathrm{SD}$ for boys. Subtle dysmorphic features that were not assigned to any known syndrome (isolated clinodactyly, micrognathia, hypertelorism, broad nasal bridge, epicanthus, low-set ears) were present in 40 subjects. Six girls additionally had disproportion of extremities (slight leg length inequality, shorter forearms, small fifth finger unilaterally). Bone age (BA) compared to the chronological age was delayed in $53 \%$ and advanced in $13 \%$ of the participants. The final height of both parents was taken into account as well. Mid-parental height (MPH) was calculated using EBMcalc Medical Calculator [21]. At the time of the study, eight participants were already treated with recombinant human growth hormone (rh-GH). For these individuals, data prior to the treatment were used. None of the participants presented with the Madelung deformity. Details are given in Supplementary File S1.

We identified altogether five alternations in the SHOX gene region including noncoding enhancer regions, of which one was present in three different subjects. Three alternations were detected by MLPA: long heterozygous deletion at least $1340 \mathrm{kbp}$ in size, long heterozygous duplication at least $324 \mathrm{kbp}$ in size, and a heterozygous duplication of the SHOX CNE- 5 enhancer element. Sanger sequencing revealed two different heterozygous alternations: a silent point variant in the exon 2 (NM_000451.3:c.63C > T) present 
in three different subjects and deletion of three codons in the noncoding region between exons 4 and 5 (NM_000451.3:c.544+15_544+23del).

\subsection{Copy Number Variants Identified by MLPA Assay}

The complete heterozygous SHOX deletion, encompassing the entire coding region together with all highly conserved non-coding DNA elements (CNEs) on both sides of the gene (except CNE-5), as well as four other genes (CRLF2, CSF2RA, IL3RA, and ASMT), was present in participant 42 (P42). The deletion is located on the Xp22-PAR1 chromosome region spanning from $\mathrm{X}: 000,380638$ to $\mathrm{X}: 001,712090$. The length of the deletion was estimated by summing the distances between the individual probes with only one copy (probe ratio 0.5 ), showing a deletion of approximately $1340 \mathrm{kbp}$.

At least $324 \mathrm{kbp}$ long heterozygous duplication, encompassing all exons and multiple regulatory elements in the SHOX region, was found in participant 75 (P75). Complete SHOX duplications appear to be extremely rare and also present in clinical conditions unrelated to ISS [22,23].

Isolated heterozygous duplication of the CNE- 5 enhancer element was identified in one female participant (P47). The duplication measured at least $364 \mathrm{bp}$ in length and did not include any SHOX coding sequences.

\subsection{Sequence Analysis of SHOX Gene}

Two different heterozygous variations were found with gene sequencing, one of which was present in three participants. According to the GnomAD population database, the frequency of silent variant M_000451.3:c.63C > T in the general population is $0.4 \%$ and was classified as benign according to the recommendation of the American College of Medical Genetics (ACMG) [24]. The frequency of the intronic deletion NM_000451.3:c.544+15_544+23del is $0.0008 \%$ and was classified as a variant of unknown significance according to the recommendation of the ACMG. Neither of these two variations affects the amino acid sequence in the protein; they are located in regions that are less important for intron excision and are also present in the general population. Therefore, it is very likely that these two variations are not disease-causing.

No CNVs, point variants, or smaller deletions/duplications were identified in the other 68 study participants.

\section{Discussion}

Determining the genetic cause of short stature in growing children is important from several points of view. It enables us to better predict a natural outcome based on the published cases (e.g., final height and possible concomitant diseases) and suggests the best treatment options (e.g., use of recombinant human growth hormone (rh-GH) in children with SHOX variations) [8].

The usual approach for detecting disease-causing variations in the SHOX region begins with determining $\mathrm{CNVs}$, especially larger deletions, as these are the most common. Point variants and small deletions or insertions are less common. The incidence of SHOX gene variants in children with ISS is currently estimated at $2-15 \%$ based on a number of screening studies $[10,25,26]$. With new, more sensitive genetic tests (e.g., MLPA) and the discovery of the importance of amplification sequences, this estimate may increase. MLPA enables the simultaneous analysis of CNVs in broad genomic regions and allows the analysis and comparison of several patients in the same experiment. It is fast, easy, and very sensitive to detect larger deletions in the gene itself and in its remote regulatory regions as well $[6,27]$. For a comprehensive diagnosis of ISS, it is important to combine different methods, from cytogenetic to molecular genetics, that enable the identification of all types of variations. In this study, we aimed to determine the frequency of genetic variations in the SHOX gene region in a group of 75 Slovenian children and young adults with ISS with two independent methods-MLPA to determine CNVs, and Sanger sequencing to identify point variants, small deletions, and insertions. 
The most clinically important finding of our study was a $1340 \mathrm{kbp}$ long deletion of the entire SHOX gene present in a boy with short stature without typical LWD clinical signs. The participant showed no signs of dysmorphism or body disproportion; however, shorter extremities and Madelung deformity were present in his mother. The mentioned deletion of the entire gene is the only undoubtedly causal variant identified in our group of short stature participants. To accurately define the breaking points of the deletion and its exact length, sequencing of the entire region should be performed. However, it is clear that the identified deletion covers the entire coding region of the SHOX gene together with all CNEs, except CNE-5, as well as four other genes (CRLF2, CSF2RA, IL3RA, and ASMT).

In addition to the $1340 \mathrm{kbp}$ long deletion, we detected two other variants with the MLPA method-at least $324 \mathrm{kbp}$ long duplication in the SHOX region and isolated duplication of the CNE-5 enhancer. In the study of SHOX/PAR1 duplications, Benito-Sanz et al. noted the presence of SHOX duplications in other clinical conditions as well, so their pathogenicity in short stature is questionable [5]. Duplication of the CNE-5 enhancer appears in the literature in combination with the deletion of the CNE-3 enhancer, which the author considers as a causal variant in the family with ISS [28]. However, there is not enough evidence for stand-alone CNE-5 duplication to be pathogenic, and further research should be undertaken. Therefore, both variants described above are considered benign.

Among the above-mentioned alternations, only complete SHOX deletion was identified as disease-causing, which represents $1.3 \%$ of our study group. Our findings therefore slightly deviate from the data from the literature, which states a $2-15 \%$ incidence of SHOX variations among individuals with ISS $[10,25,26]$. The outcome of the analysis is significantly influenced by the formulation of inclusion and exclusion criteria. The most basic criteria for creating a cohort group, also used in our study, are growth retardation (height below -2 SD) and no specific known causative disorders. Differences between the criteria occur in the inclusion/exclusion of patients with dysmorphic signs and also regarding the age of the patients since some authors include only prepubertal children, while others include persons up to 29 years of age. The correct interpretation of the clinical definition of short stature is crucial and enables the most homogeneous study group. Diversity in diagnostic yield is also due to the use of various methodological approaches in studies used to detect different types of variants. The changes may thus be located in regions that are not visible with the approach used. Nevertheless, we carefully selected a homogenous group of patients with ISS and applied all appropriate genetic tests to detect causal variants inside the SHOX gene and enhancer region.

In addition to variants in the SHOX gene itself, enhancer sequences are also of great importance and, when altered, they may contribute to the development of growth failure. This was demonstrated by Chen et al. in their study of 735 participants with ISS and 58 Leri-Weill syndrome patients using the FISH and MLPA methods. In the ISS group, the presence of $\mathrm{CNV}$ in the $\mathrm{SHOX}$ region was confirmed in 31 individuals $(4.2 \%), 8$ of whom $(26 \%)$ carried the microdeletion only in enhancer sequences positioned at least $150 \mathrm{~kb}$ away from the coding region. In Leri-Weill syndrome patients, 29 microdeletions were identified, 13 of which (45\%) involved only enhancer sequences. In all these cases, the SHOX gene remained intact [10]. In the cohort of French children with ISS, Rosilio et al. identified SHOX deficiency in $16.9 \%$, more than half of which had a deletion downstream of the gene in the enhancer region [7]. This confirms that enhancer deletions in the SHOX region are a relatively common cause of short stature in children diagnosed with ISS. In this region, a total of seven conserved non-coding elements have been identified that act as enhancer elements for the SHOX gene transcription. Four of them (CNE4, CNE5, ECR1, CNE9/ECS4) are located downstream of the gene, and three of them (CNE-5, CNE-3, CNE-2) upstream of the gene. The loss of enhancer elements leads to reduced gene expression and consequently affects the growth and development of the individual $[28,29]$.

The results of this research are consistent with a similar study in the Slovenian population conducted by Hovnik et al. in 2015 at the University Children's Hospital of Ljubljana. The SHOX region was analysed by fluorescence in situ hybridization to identify large 
deletions, and direct DNA sequencing was used to identify point variants and small deletions or insertions. They concluded that small or large-scale SHOX alternations are not a common cause of short stature among Slovenian children with ISS, as they did not find any variations in the SHOX gene in a group of 40 participants of the study [27].

In conclusion, despite the integrated genetic approach, using MLPA analysis and Sanger sequencing, our study results indicate that SHOX variants are not a common cause of ISS in Slovenian patients. Further research and functional studies are needed to confirm the pathogenicity of different SHOX variants, especially duplications. A definite genetic diagnosis also enables a more accurate prognosis and the possibility of treatment. In prepubertal children with ISS due to SHOX deficiency, an increase in final height by 7 to $10 \mathrm{~cm}$ can be achieved by rh-GH therapy [30].

Supplementary Materials: The following are available online at https:/ / www.mdpi.com/article/10 .3390 /genes12101546/s1, Table S1: Detailed features of all participants with ISS.

Author Contributions: Conceptualization, T.H. and K.T.P.; methodology, T.H. and L.S.; formal analysis, J.S. and M.F.; investigation, J.S. and L.S.; data curation, J.S. and L.S.; writing-original draft preparation, J.S.; writing—review and editing, T.H., K.T.P., L.S., M.F. and P.K.; supervision, T.H. and T.B.; funding acquisition, T.B. All authors have read and agreed to the published version of the manuscript.

Funding: This work was supported in part by the Slovenian Research Agency (grant no. P3-0343) and tertiary research project no. TP 20170122.

Institutional Review Board Statement: The study was conducted according to the guidelines of the Declaration of Helsinki and approved by the Slovene Medical Ethics Committee (0120-36/2019/4).

Informed Consent Statement: Written informed consent was obtained from all subjects involved in the study.

Acknowledgments: We thank Jernej Kovač, Tine Tesovnik, Maruša Debeljak, Anja Vujić, Ana Grom, Vanda Cucek, Sončka Jazbinšek, and all other coworkers at the Clinical Institute of Special Laboratory Diagnostics for their expert technical assistance.

Conflicts of Interest: The authors declare no conflict of interest.

\section{References}

1. Murray, P.; Clayton, P.; Chernausek, S.D. A genetic approach to evaluation of short stature of undetermined cause. Lancet Diabetes Endocrinol. 2018, 6, 564-574. [CrossRef]

2. Barstow, C.; Rerucha, C. Evaluation of Short and Tall Stature in Children. Am. Fam. Physician 2015, 92.

3. Cohen, P.; Rogol, A.; Deal, C.L.; Saenger, P.; Reiter, E.O.; Ross, J.L.; Chernausek, S.D.; Savage, M.O.; Wit, J.M. Consensus Statement on the Diagnosis and Treatment of Children with Idiopathic Short Stature: A Summary of the Growth Hormone Research Society, the Lawson Wilkins Pediatric Endocrine Society, and the European Society for Paediatric Endocrinology Workshop. J. Clin. Endocrinol. Metab. 2008, 93, 4210-4217. [CrossRef] [PubMed]

4. Guo, M.H.; Hirschhorn, J.; Dauber, A. Insights and Implications of Genome-Wide Association Studies of Height. J. Clin. Endocrinol. Metab. 2018, 103, 3155-3168. [CrossRef] [PubMed]

5. Benito-Sanz, S.; Barroso, E.; Suñer, D.H.; Hisado-Oliva, A.; Romanelli, V.; Rosell, J.; Aragones, A.; Caimari, M.; Argente, J.; Ross, J.L.; et al. Clinical and Molecular Evaluation of SHOX/PAR1 Duplications in Léri-Weill Dyschondrosteosis (LWD) and Idiopathic Short Stature (ISS). J. Clin. Endocrinol. Metab. 2011, 96, E404-E412. [CrossRef] [PubMed]

6. Binder, G. Short Stature due to SHOX Deficiency: Genotype, Phenotype, and Therapy. Horm. Res. Paediatr. 2011, 75, 81-89. [CrossRef] [PubMed]

7. Rosilio, M.; Huber, C.; Sapin, H.; Carel, J.-C.; Blum, W.F.; Cormier-Daire, V. Genotypes and Phenotypes of Children with SHOX Deficiency in France. J. Clin. Endocrinol. Metab. 2012, 97, E1257-E1265. [CrossRef]

8. Blum, W.F.; Ross, J.L.; Zimmermann, A.G.; Quigley, C.A.; Child, C.J.; Kalifa, G.; Deal, C.; Drop, S.L.; Rappold, G.; Cutler, G.B., Jr. GH Treatment to Final Height Produces Similar Height Gains in Patients With SHOX Deficiency and Turner Syndrome: Results of a Multicenter Trial. J. Clin. Endocrinol. Metab. 2013, 98, E1383-E1392. [CrossRef]

9. Institute of Medical Genetics in Cardiff. HGMD®Gene Result. The Human Gene Mutation Database [Internet]. Available online: http:/ / www.hgmd.cf.ac.uk/ac/index.php (accessed on 25 August 2021).

10. Chen, J.; Wildhardt, G.; Zhong, Z.; Roth, R.; Weiss, B.; Steinberger, D.; Decker, J.; Blum, W.F.; A Rappold, G. Enhancer deletions of the SHOX gene as a frequent cause of short stature: The essential role of a $250 \mathrm{~kb}$ downstream regulatory domain. J. Med. Genet. 2009, 46, 834-839. [CrossRef] 
11. Fukami, M.; Seki, A.; Ogata, T. SHOX Haploinsufficiency as a Cause of Syndromic and Nonsyndromic Short Stature. Mol. Syndr. 2016, 7, 3-11. [CrossRef]

12. Pedicelli, S.; Peschiaroli, E.; Violi, E.; Cianfarani, S. Controversies in the Definition and Treatment of Idiopathic Short Stature (ISS). J. Clin. Res. Pediatr. Endocrinol. 2011, 1, 105-115. [CrossRef]

13. Ranke, M.B. Growth Hormone Deficiency: Diagnostic Principles and Practice. 2011, pp. 102-137. Available online: https: / / www.karger.com/Article/Abstract/327405 (accessed on 20 September 2019). [CrossRef]

14. Thodberg, H.H.; Kreiborg, S.; Juul, A.; Pedersen, K.D. The BoneXpert Method for Automated Determination of Skeletal Maturity. IEEE Trans. Med. Imaging 2008, 28, 52-66. [CrossRef] [PubMed]

15. Cole, T.J. The LMS method for constructing normalized growth standards. Eur. J. Clin. Nutr. 1990, 44, 45-60. [PubMed]

16. Qiagen. FlexiGene DNA Handbook: For Purification of DNA from Human Whole Blood, Buffy Coat, Cultured Cells. Available online: https:/ / www.qiagen.com/us/products/discovery-and-translational-research/dna-rna-purification/dna-purification/ genomic-dna/flexigene-dna-kit/ (accessed on 25 August 2021).

17. MRC-Holland. MLPA®General Protocol: Instructions For Use. Available online: https://www.mrcholland.com/products/4838 /MLPAGeneralProtocolMDP-v007.pdf (accessed on 18 March 2019).

18. MRC-Holland. Product Description SALSA®MLPA®Probemix P018-G2 SHOX. Available online: https://www.mrcholland.com/ products /27527/ProductdescriptionP018-G2SHOX-v04.pdf (accessed on 25 August 2021).

19. MRC-Holland. Coffalyser.Net Reference Manual. Available online: https://support.mrcholland.com/downloads/files/ coffalyser-net-reference-manual. (accessed on 25 August 2021).

20. FigTree v1.4.2. Available online: https:/ / blast.ncbi.nlm.nih.gov/Blast.cgi (accessed on 16 February 2021).

21. EBMcalc Medical Calculator. Available online: https://ebmcalc.com/HeightPotential.htm. (accessed on 21 September 2021).

22. Thomas, N.S.; Harvey, J.F.; Bunyan, D.J.; Rankin, J.; Grigelioniene, G.; Bruno, D.L.; Tan, T.Y.; Tomkins, S.; Hastings, R. Clinical and molecular characterization of duplications encompassing the human SHOX gene reveal a variable effect on stature. Am. J. Med. Genet. Part A 2009, 149A, 1407-1414. [CrossRef] [PubMed]

23. Fukami, M.; The Japanese SHOX study group; Naiki, Y.; Muroya, K.; Hamajima, T.; Soneda, S.; Horikawa, R.; Jinno, T.; Katsumi, M.; Nakamura, A.; et al. Rare pseudoautosomal copy-number variations involving SHOX and/or its flanking regions in individuals with and without short stature. J. Hum. Genet. 2015, 60, 553-556. [CrossRef]

24. Richards, S.; Aziz, N.; Bale, S.; Bick, D.; Das, S.; Gastier-Foster, J.; Grody, W.W.; Hegde, M.; Lyon, E.; Spector, E.; et al. Standards and guidelines for the interpretation of sequence variants: A joint consensus recommendation of the American College of Medical Genetics and Genomics and the Association for Molecular Pathology. Genet. Med. 2015, 17, 405-423. [CrossRef] [PubMed]

25. Rappold, G.A.; Fukami, M.; Niesler, B.; Schiller, S.; Zumkeller, W.; Bettendorf, M.; Heinrich, U.; Vlachopapadoupoulou, E.; Reinehr, T.; Onigata, K.; et al. Deletions of the Homeobox GeneSHOX(Short Stature Homeobox) Are an Important Cause of Growth Failure in Children with Short Stature. J. Clin. Endocrinol. Metab. 2002, 87, 1402-1406. [CrossRef] [PubMed]

26. Hirschfeldova, K.; Solc, R.; Baxova, A.; Zapletalova, J.; Kebrdlova, V.; Gaillyova, R.; Prasilova, S.; Soukalova, J.; Mihalova, R.; Lnenicka, P.; et al. SHOX gene defects and selected dysmorphic signs in patients of idiopathic short stature and Léri-Weill dyschondrosteosis. Gene 2012, 491, 123-127. [CrossRef] [PubMed]

27. Hovnik, T.; Schweiger, D.; Kotnik, P.; Kovač, J.; Battelino, T.; Podkrajsek, K.T. Cytogenetic and Molecular Genetic Characterization of Children with Short Stature / Citogenetska in Molekularno Genetska Opredelitev Nizke Rasti Pri Otrocih. Slov. J. Public Health 2015, 54, 98-102. [CrossRef]

28. Bunyan, D.J.; Baffico, M.; Capone, L.; Vannelli, S.; Iughetti, L.; Schmitt, S.; Taylor, E.-J.; Herridge, A.A.; Shears, D.; Forabosco, A.; et al. Duplications upstream and downstream of SHOX identified as novel causes of Leri-Weill dyschondrosteosis or idiopathic short stature. Am. J. Med. Genet. Part A 2015, 170, 949-957. [CrossRef] [PubMed]

29. Marchini, A.; Ogata, T.; Rappold, G.A. A Track Record on SHOX: From Basic Research to Complex Models and Therapy. Endocr. Rev. 2016, 37, 417-448. [CrossRef] [PubMed]

30. Binder, G.; Rappold, G.A. SHOX Deficiency Disorders-GeneReviews ${ }^{\circledR} —$ NCBI Bookshelf. GeneReviews(®). Available online: https:/ / www.ncbi.nlm.nih.gov/books/NBK1215/ (accessed on 25 August 2021). 